\title{
SAOCOM 1A INTERFEROMETRIC ERROR MODEL AND ANALYSIS
}

\author{
Pablo Andrés Euillades ${ }^{(1)}$, Leonardo Daniel Euillades ${ }^{(1)}$, Mario Azcueta ${ }^{(2)}$, Gustavo Sosa ${ }^{(1)}$ \\ (1) Instituto CEDIAC - FI - UNCuyo \& CONICET, Centro Universitario, Mendoza (5500), Argentina, \\ Email:\{peuillades, leuillades, gsosa\}@cediac.uncu.edu.ar \\ (2) CONAE, Av. Paseo Colón 751, CABA (1063),Argentina,Email: mazcueta@conae.gov.ar
}

\begin{abstract}
This article addresses an analysis of the precision attainable with SAOCOM satellites for DInSAR applications. Error model considers orbital, topographic, and atmospheric residual phase terms. It also estimates geometric, doppler, thermal and temporal decorrelation. Results relevant for the Stripmap 7 Dual Polarization mode are deeply discussed, and summarized error metrics for all stripmap modes are presented. The study concludes that SAOCOM sensors will be capable of resolving a good number of real deformation patterns.
\end{abstract}

\section{INTRODUCTION}

The SAOCOM mission, being developed by CONAE from Argentina (Comision Nacional de Actividades Espaciales) is an earth observation system composed of two identical satellites (SAOCOM 1A and SAOCOM 1B) flying in constellation, carrying each one a fully polarimetric Synthetic Aperture Radar (SAR) operating in L-band.

The two SAOCOM satellites will fly in a Low-Earth Orbit at $620 \mathrm{~km}$ altitude, providing 16-day revisit-time for single-pass acquisitions and 8-day revisit-time with both SAOCOM satellites. They will also be in constellation with 4 Italian COSMO Sky-Med satellites.

It is in the interest of CONAE to provide interferometry products to final users, by exploiting SAOCOM Stripmap acquisitions. Hence, a mathematical model was developed to assess the expected accuracy of interferometry products, considering multiple error sources (orbit knowledge error, decorrelation noise, DEM errors, etc.)

In this work, we address the suitability of the SAOCOM system to estimate surface deformation mapping. We present the error model developed for estimating the LOS displacement variance given the SAOCOM Stripmap acquisition modes characteristics, and discuss some of the obtained results.

\section{ERROR MODEL}

In absence of other phase components, the relationship between interferometric phase and Line-of-Sight displacement is given by [1]:

$$
d_{L O S}=\frac{\lambda}{4 \pi} \Delta \phi^{D I n S A R}
$$

In a real case, the interferometric phase contains information related with the acquisition geometry $\left(\Delta \phi^{\text {orbit }}\right)$, the topography of the illuminated area $\left(\Delta \phi^{\text {topo }}\right)$, soil displacement produced between S1 and S2 acquisition times $\left(\Delta \phi^{\text {defo }}\right)$, differences in the atmospheric and/or ionospheric state at the time of S1 and $\mathrm{S} 2$ acquisitions $\left(\Delta \phi^{\text {defo }}\right)$, and decorrelation noise $\left(\Delta \phi^{\text {noise }}\right)$. The differential phase at a point of coordinates $(x, R)$ in range and azimuth can be expressed, after topographic, orbital and atmospheric phase compensation, as:

$$
\begin{gathered}
\Delta \phi^{\text {DInSAR }}=E\left\{\Delta \phi^{\text {deformation }}\right\}+ \\
+\Delta \phi^{\text {res orbit }}+\Delta \phi^{\text {res topo }}+ \\
+\Delta \phi^{\text {res atm }}+\Delta \phi^{\text {noise }}
\end{gathered}
$$

where residual terms account for uncertainty in estimating the true orbital, topographic an atmospheric phase contributions present in the original interferogram.

Furthermore, in a practical case LOS deformation observations are obtained by integrating spatial gradients between resolution cells. In other words, deformation at one generic point $p$ of coordinates $\left(x_{p}, R_{p}\right)$ is relative to another point $q$ of coordinates $\left(x_{q}, R_{q}\right)$ located inside the processed scene. As a consequence, the estimated relative LOS displacement between $p$ and $q$ as a function of the relative interferometric phase change is:

$$
\begin{gathered}
\hat{d}_{p q}^{L O S}=\frac{\lambda}{4 \pi} \cdot \Delta \Phi_{p q}^{D I n S A R}= \\
=\frac{\lambda}{4 \pi} \cdot E\left\{\Delta \phi_{p q}^{\text {defo }}\right\}+\frac{\lambda}{4 \pi} \cdot \Delta \phi_{p q}^{\text {res }} \text { orbit }+ \\
+\frac{\lambda}{4 \pi} \cdot \Delta \phi_{p q}^{\text {restopo }}+\frac{\lambda}{4 \pi} \cdot \Delta \phi_{p q}^{\text {atm }}+\frac{\lambda}{4 \pi} \cdot \Delta \phi_{p q}^{\text {noise }}
\end{gathered}
$$

where $\Delta \phi_{p q}$ is short notation for $\Delta \phi_{p}-\Delta \phi_{q}$.

From previous equation, and assuming that all terms are uncorrelated, LOS displacement variance is: 


$$
\begin{gathered}
\sigma_{\hat{d}_{p q}^{L O S}}^{2}=\frac{\lambda}{4 \pi} \cdot \sigma_{\Delta \phi_{p q}^{\text {res orbit }}}^{2}+ \\
+\frac{\lambda}{4 \pi} \cdot \sigma_{\Delta \phi_{p q}^{\text {res topo }}}^{2}+\frac{\lambda}{4 \pi} \cdot \sigma_{\Delta \phi_{p q}^{2}}^{2}+\frac{\lambda}{4 \pi} \cdot \sigma_{\Delta \phi_{p q}^{\text {noise }}}^{2}
\end{gathered}
$$

Residual orbital variance $\left(\sigma_{\Delta \phi_{p q}^{\text {res orbit }}}^{2}\right)$ can be estimated by considering that the perpendicular baseline is known except for an error $\Delta B_{\perp}$. Under this assumption, residual orbital variance between two points located at ranges $p$ and $q$ can be expressed as a function of perpendicular baseline uncertainty as:

$$
\begin{gathered}
\sigma_{\Delta \phi_{r p q}^{\text {res orbit }}}^{2}= \\
=\left[\frac{4 \pi}{\lambda} \int_{r_{p}}^{r_{q}} \frac{1}{r \cdot \sin \vartheta_{0}}\left(\cos \vartheta_{0}-\frac{r}{R_{E}+H}\right) \cdot d r\right]^{2} . \\
\cdot \sigma_{B \text { perp }}^{2}
\end{gathered}
$$

where $\vartheta_{0}$ is the look angle to the ellipsoid, $R_{E}$ is the Earth's radius and $H$ is the orbital height.

Topographic residual term, $\left(\sigma_{\Delta \phi_{p q}^{2}}^{\text {res topo }}\right)$, is estimated by considering the expression derived in a previous work [2]. Uncertainty sources are the baseline error $\left(\Delta B_{\perp}\right)$ and the DEM error $(\Delta z)$. Residual topography variance is expressed as a function of the perpendicular baseline uncertainty and DEM errors at point $p$ and $q$ :

$$
\begin{gathered}
\sigma_{\Delta \phi_{p q}^{\text {res topo }}}^{2}= \\
=\left[\frac{4 \pi B_{\text {perp }}}{\lambda} \frac{1}{r_{p} \sin \vartheta_{p}}\right]^{2} \cdot \sigma_{\mathrm{z}_{\mathrm{p}}}^{2} \\
+\left[\frac{4 \pi B_{\text {perp }}}{\lambda} \frac{1}{r_{q} \sin \vartheta_{q}}\right]^{2} \cdot \sigma_{\mathrm{z}_{\mathrm{q}}}^{2} \\
-\left[\frac{4 \pi B_{\text {perp }}}{\lambda}\right]^{2} \frac{2}{\mathrm{r}_{\mathrm{p}} \mathrm{r}_{\mathrm{q}} \sin \vartheta_{\mathrm{p}} \sin \vartheta_{\mathrm{q}}} \cdot \sigma_{\mathrm{z}_{\mathrm{p}} \mathrm{z}_{\mathrm{q}}} \\
+\left[\frac{4 \pi \mathrm{z}_{\mathrm{q}}}{\lambda r_{q} \sin \vartheta_{q}}\right]^{2} \sigma_{\mathrm{B}_{\mathrm{perp}}}^{2}
\end{gathered}
$$

For estimating the atmospheric contributions we adopted the model proposed in [3]:

$$
\sigma_{p q}^{a t m}[m m]=c L^{\alpha}+k \cdot H
$$

where $L$ is the distance between $p$ and $q$ points in $\mathrm{km}, H$ is the height change in meters, $c, \alpha$ and $k$ are parameters depending on the site location and time between scenes.

Decorrelation variance have been estimated by numerically integrating the interferometric phase probability density function for distributed targets [4]. In order to relate the SAOCOM operating parameters with coherence we decomposed the last in the following terms:

$$
\begin{gathered}
\gamma= \\
=\gamma_{\text {thermal }} \cdot \gamma_{\text {geom }} \cdot \gamma_{\text {doppler }} \cdot \gamma_{\text {proc }} \cdot \gamma_{\text {temporal }}
\end{gathered}
$$

Thermal decorrelation term $\left(\gamma_{\text {thermal }}\right)$ have been estimated as $\gamma_{\text {thermal }}=\frac{1}{1+S N R^{-1}}$, following Curlander and McDonough [5]. Geometric term $\left(\gamma_{\text {geom }}\right)$ is derived from the linear model presented by Zebker and Villasenor [6]. Doppler term $\left(\gamma_{\text {doppler }}\right)$ was computed following the linear model presented in Hanssen [7]. Processing term was computed from values provided by CONAE. Temporal decorrelation do not depend on the system settings, so we considered a fixed value.

\section{RESULTS}

We implemented the described error model in a Python programmed application. A plain text parameter file provides an easy way for setting acquisition geometry parameters like SAOCOM Stripmap mode, perpendicular baseline, perpendicular baseline error and parallel baseline rate error; terrain parameters like temporal coherence, slope, height change; and processing parameters like filtering, DEM error and multilooking factors. Satellite operating parameters are taken from a

\begin{tabular}{|c|c|}
\hline \multicolumn{2}{|l|}{ Acquisition mode: DP7 } \\
\hline Polarization & Dual-Pol \\
\hline Look angle [deg] & $39.6-41.9 \mathrm{deg}$ \\
\hline NESZ & $-35 \mathrm{~dB}$ \\
\hline $\begin{array}{llll}\begin{array}{l}\text { Resolution } \\
\text { azimuth) }\end{array} & \text { (ground } & \text { range } & \\
\end{array}$ & $5.54 \mathrm{~m} \times 8.5 \mathrm{~m}$ \\
\hline Ground range & $48 \mathrm{~km}$ \\
\hline
\end{tabular}
database which contains all the relevant information like carrier frequency, bandwidths, PRF, sampling frequency, look angle, etc.

With this tool we estimated the expected LOS displacement errors along a range line for SAOCOM Stripmap acquisition modes. We present here the results for acquisition mode DP7, which is representative of the performance achievable with the rest of the SAOCOM Stripmap acquisition modes. The main characteristics of DP7 mode are summarized in Tab. 1.

Table 1. Stripmap Mode 7 DP characteristics. 


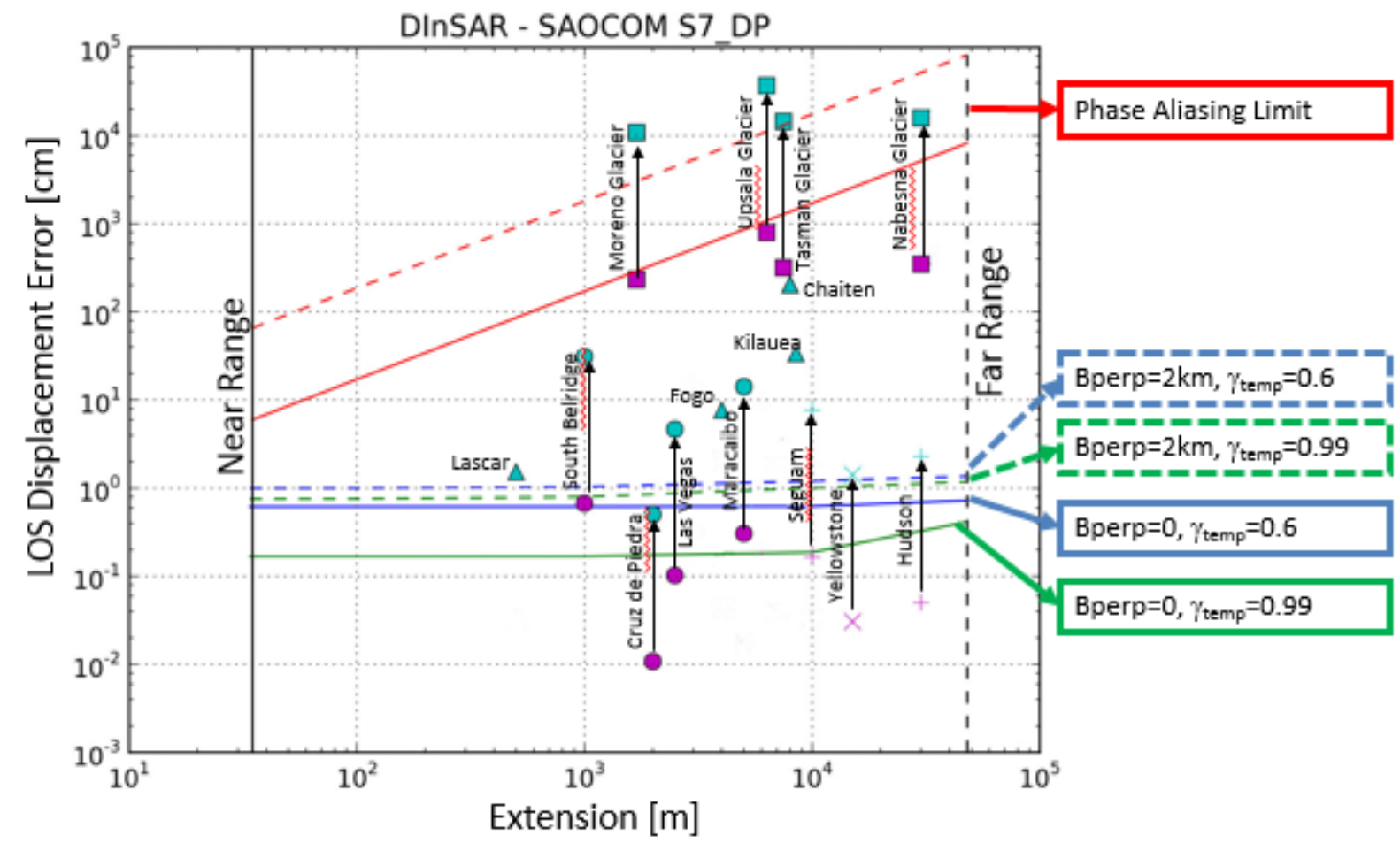

Figure 1. Stripmap mode 7 dual polarization (S7 DP) for Base Scenario. Error metrics are represented by blue and green lines, as indicated in the chart. Red lines at the top represent the phase aliasing limit for multilook process (solid) and full resolution process (dashed). Real cases included within the chart are subsidence (dots) after 8 days and a year, volcanic eruptions (triangles), pre- and inter-eruptive volcanic deformation (plus, crosses) and glacier movement (squares).

We computed error metrics by defining two extreme scenarios. First one, named Base Scenario is a low error one intended for estimating the minimum achievable precision on displacements between near and far range. Parameters defining the Base Scenario are perpendicular baseline error $\sigma B$ perp $=0.1 \mathrm{~m}$, multilooking factors of $10 \times 10$ (slant range dimension of $25 \mathrm{~m}$, azimuth dimension of $35 \mathrm{~m}$ ), processing includes spectral filtering, DEM error $\sigma_{z}=3 \mathrm{~m}$, flat terrain (slope $=0$, height change $=0 \mathrm{~m}$ ), coherence due to processing errors is 0.95 and coherence due to thermal effects is $\sim 0.98$. Atmospheric effects are not considered.

Base scenario results are shown in Fig. 1. The horizontal axis shows the characteristic spatial width of the imaging system, and ranges between $25 \mathrm{~m}$ (black solid line) and $48 \mathrm{~km}$ (black dashed line) that is the swath width for the investigated acquisition mode. Vertical axis show the measurable LOS deformation. Solid lines at the bottom represent the LOS deformation standard deviation computed by considering a null perpendicular baseline and temporal coherence of 0.99 (green) and 0.6 (blue). Dashed lines are obtained by setting the perpendicular baseline to $2000 \mathrm{~m}$, temporal coherence to 0.99 (green) and 0.6 (blue). All those lines represent the system's precision under different circumstances. Red lines at the figure's top represent the maximum LOS change measurable without phase aliasing. Dashed line is computed considering full-resolution processing, i.e. unwrapping the full resolution interferogram, whereas solid line is computed by processing at the selected multilooking factors.

Second scenario is called Realistic Scenario. It is computed by using essentially the same parameter settings as for the Base Scenario but including atmospheric effects computed from Eq. 7 with $c=2.5$, $\alpha=0.5$ and $k=0$. Terrain profile in this case considers a height change of $3600 \mathrm{~m}$ between near and far range and varying local slopes between $0-14$ degrees. Results are shown in Fig. 2.

From Figs. 1 and 2 it is clear that, in absence of atmospheric conditions change between acquisitions the error is well under the $\mathrm{cm}$ for temporal coherence as low as 0.6 and null perpendicular baseline. When perpendicular baseline is $2 \mathrm{~km}$ long, the error is about one $\mathrm{cm}$ in the near range and increases up to $1.4 \mathrm{~cm}$ at far range due to the baseline uncertainty. When atmospheric conditions are considered, the error increases at far range until reaching almost $3 \mathrm{~cm}$. It is clear from Fig. 2 that the atmospheric contribution becomes dominant with respect to orbital uncertainty and coherence loss. 


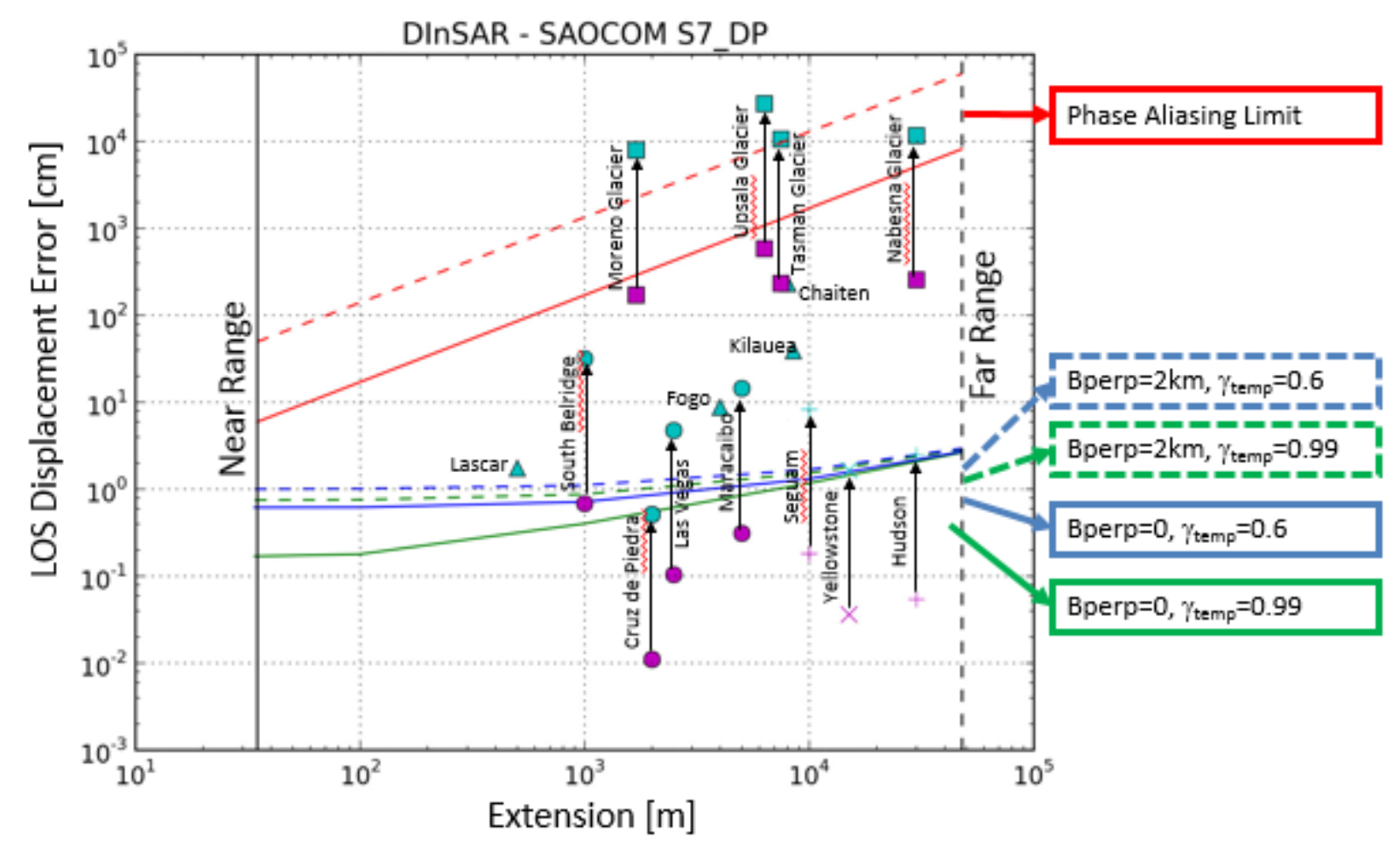

Figure 2. Stripmap mode 7 dual polarization (S7 DP) for Realistic Scenario. Error metrics are represented by blue and green lines, as indicated in the chart. Red lines at the top represent the phase aliasing limit for multilook process (solid) and full resolution process (dashed). Real cases included within the chart are subsidence (dots) after 8 days and a year, volcanic eruptions (triangles), pre-and inter-eruptive volcanic deformation (plus, crosses) and glacier movement (squares).

Summarizing, in a very favourable situation (solid green line in Fig. 1) system's precision is about $2 \mathrm{~mm}$. Error increasing at far range is due to the perpendicular baseline error which becomes more significant as distance from the reference point (near range) increases. However, considering a temporal coherence of 0.6 (solid blue line), precision below $1 \mathrm{~cm}$ is achievable. With increasing baseline, precision degrades to between 1 and $2 \mathrm{~cm}$ depending on temporal coherence (dashed lines). Note that for baselines between $0 \mathrm{~m}$ (uncommon) and $2000 \mathrm{~m}$ and good coherence the precision always maintains sub-centimeter.

Fig. 3 displays a similar analysis carried out for all stripmap modes, both dual polarization and quad polarization. In this case, separation between $\mathrm{p}$ and $\mathrm{q}$ points within the differential interferogram is $10 \mathrm{~km}$. Blue dots are the error for the base scenario, whereas red ones represent the error in the realistic scenario. We also included the analysis of an intermediate scenario with temporal coherence of 0.75 , relatively moderate terrain profile (height change of $300 \mathrm{~m}$ and low local slopes) and $1 \mathrm{~km}$ perpendicular baseline. Black bars are the errors ranging from atmospheric-free (bottom) to atmosphericconsidered (top). We consider that this scenario is more probable in most real cases than the extreme ones.
From the figure, it is clear that modes S5DP to S9DP are those more suitable for differential interferometric applications, being the expected error between 0.5 and $1.4 \mathrm{~cm}$.

\section{CONCLUSION}

A mathematical model was presented to assess the surface deformation mapping accuracy achievable by processing a single interferometric pair. The developed model is general enough to account for multiple error sources and multiple sensors characteristics. SAOCOM Stripmap acquisition parameters were particularly considered to compute the final errors.

As a conclusion of the analysis, we can say that SAOCOM system, although its main objective is soil moisture characterization, has also good interferometric capabilities. As shown by our results, it is capable of resolving a good number of real deformation patterns. Taken into account that the shown simulations only consider one image pair, even better error metrics are expected from time-series processing. 


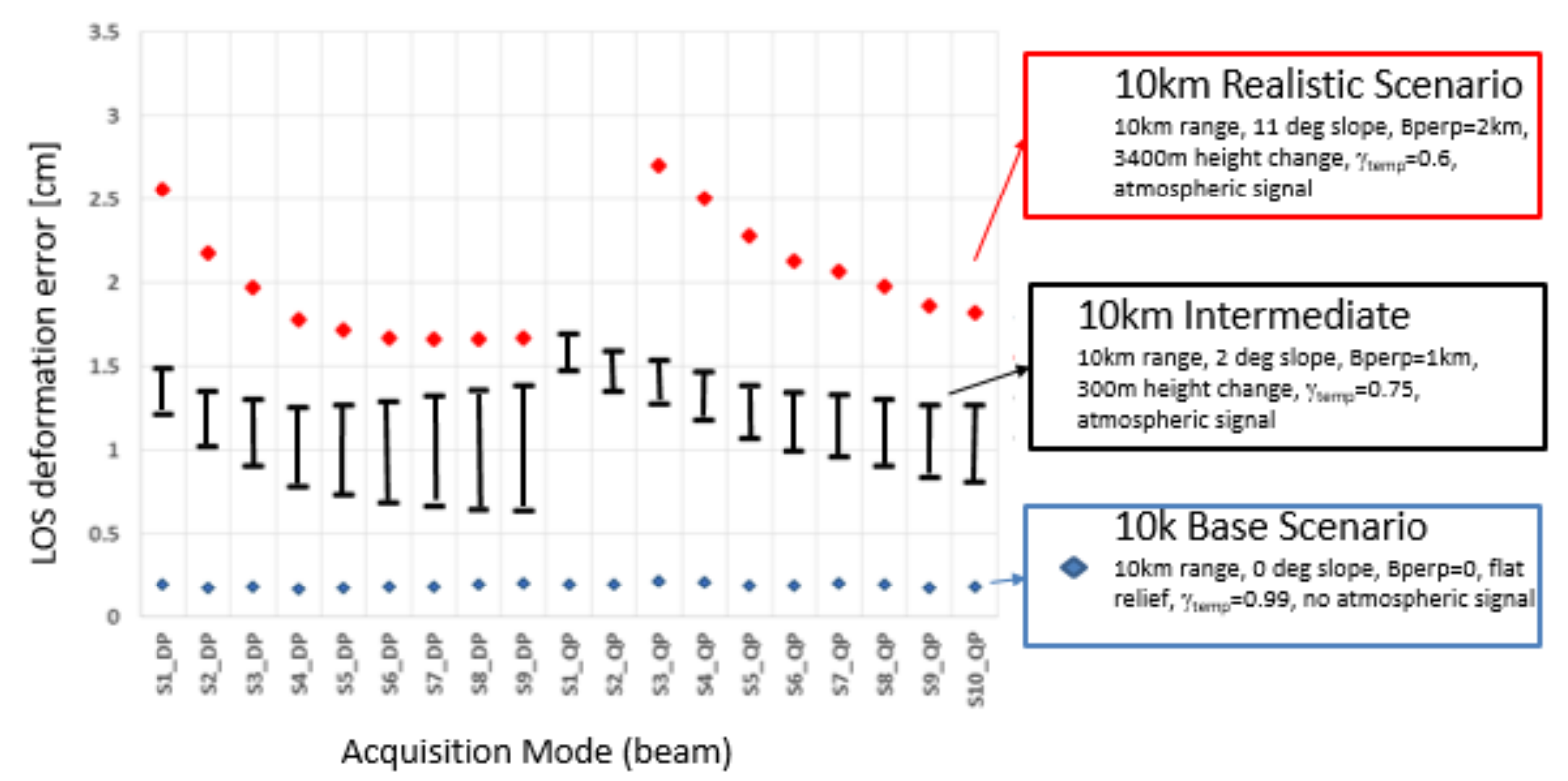

Figure 3. Error metrics for all stripmap modes of SAOCOM for two points separated by $10 \mathrm{~km}$ in ground range. Blue dots represent the low error scenario, red ones represent the realistic scenario. Black bars represent an intermediate scenario without and with atmospheric effects.

\section{REFERENCES}

1. Zebker, H., Rosen, P.A., \& Hensley, S., (1997). Atmospheric effects in interferometric synthetic aperture radar surface deformation and topographic maps. J. Geophys. Res. 102(B4), 7547-7563

2. Pepe, A., (2006). Advanced differential Interferometric SAR techniques, Chapter 1: SAR Fundamentals. $\mathrm{PhD}$ Thesis. Universita' degli Studi di Napoli "Federico II"

3. Emardson, T.R, Simons, M. \& Webb, F.H. (2003). Neutral atmospheric delay in interferometric synthetic aperture radar applications: Statistical description and mitigation. J. Geophys. Res.108(B5), ETG 1-8

4. Bamler, R., \& Hartl, P. (1998) Synthetic Aperture Radar Interferometry, Inverse Problems, 14, R1R54.

5. Curlander, J.C. \& McDonough, R.N., (1991). Synthetic Aperture Radar. Systems and Signal Processing. New York: John Wiley \& Sons.

6. Zebker, H.A. \& Villasenor, J., (1992) Decorrelation in interferometric radar echoes. IEEE TGARS. 30(5). 950-959.

7. Hanssen, R.F. (2001). Radar Interferometry - Data
Interpretation and Error Analysis. Kluwer Academic Publishers. 